\title{
Análisis critico de discurso y etnografía: Una propuesta metodológica para el estudio de la alteridad con poblaciones migrantes
}

\author{
Critical discourse analysis and ethnography: A methodological \\ proposal for the study of otherness with migrant populations
}

\author{
Antonia Olmos Alcaraz \\ Universidad de Granada \\ antonia@ugr.es (ESPAÑA)
}

\author{
Recibido: 14.10 .2014 \\ Aceptado: 01.09.2015
}

\section{RESUMEN}

El artículo muestra la propuesta metodológica desarrollada en un trabajo de tesis doctoral que lleva por título "La población inmigrante extranjera y la construcción de la diferencia. Discursos de alteridad en el sistema educativo andaluz" (Olmos Alcaraz 2009). Se analiza la combinación de Análisis Crítico de Discurso y de trabajo etnográfico, para abordar el estudio de la construcción de alteridad hacia el alumnado inmigrado de nacionalidad extranjera en España. Para ello se describe el proceso de producción y análisis de los datos y los resultados obtenidos en la investigación en el plano metodológico, abogando con ello por enfoques multi, inter y transdiciplinares dentro de las Ciencias Sociales.

\section{PALABRAS CLAVE}

Análisis Crítico de Discurso, Etnografía, Migraciones, Alteridad.

\begin{abstract}
The paper shows a methodological proposal carried on in a $\mathrm{PhD}$ research, entitled "La población inmigrante extranjera y la construcción de la diferencia. Discursos de alteridad en el sistema educativo andaluz" (Olmos Alcaraz 2009). In the text is analized the convination of Critical Discourse Analysis and Ethno-
\end{abstract}


graphy, to approach the study of the 'construction of the difference' toward foreign migrant students in Spain. To do that in the paper are described the procceses of production and analysis of the data and the results obtained in the research on the methodological sphere, to claim an inter, multi and trans-disciplinary approach in Social Science

\section{KEY WORDS}

Critical Discourse Analysis, Ethnography, Migrations, Otherness.

\section{INTRODUCCIÓN}

El presente texto describe la propuesta metodológica desarrollada en mi trabajo de tesis doctoral, titulado La población inmigrante extranjera y la construcción de la diferencia. Discursos de alteridad en el sistema educativo andaluz, donde el objetivo era estudiar la construcción de la diferencia, en tanto que proceso social y cultural, con respecto al alumnado inmigrado de nacionalidad extranjera en España.

En el diseño de dicho trabajo de investigación fue fácil decidir el paradigma metodológico en el que ubicarme, siendo la investigación cualitativa el enfoque elegido dado su "énfasis en el [estudio del] lenguaje, en la interpretación de los hechos humanos y la toma del punto de vista del actor" (Alvira 1983: 54), lo que me permitía abordar convenientemente mi objeto teórico de estudio. No fue tan sencillo, sin embargo, situarme en una única metodología, porque mi investigación se vislumbraba desde el principio como multi, inter y transdiciplinar ${ }^{1}$. Entiendo que la realidad social es demasiado compleja para ser estudiada desde una sola disciplina y metodología, por lo que es muy pertinente aprovechar los logros que se dan desde distintas aproximaciones. De igual manera consagrar metodologías a disciplinas me parece limitador, por lo que estimo conveniente y necesario la combinación y la puesta en práctica de la pluralidad metodológica dentro de la investigación social.

Por todo ello, y para responder de la manera más holista posible al abordaje de las relaciones de alteridad producidas alrededor del alumnado inmigrado de nacionalidad extranjera, opté por realizar Análisis Crítico de Discurso (en adelante ACD) y trabajo de campo etnográfico, para abordar lo que para mí eran discursos ${ }^{2}$ de alteridad de "políticos, políticas y escuela" (Olmos Alcaraz 2010).

1 Las más de las veces estos tres términos son tomados como sinónimos, no obstante presentan particularidades importantes que tienen que ver con el objetivo último de la colaboración entre disciplinas. Para un análisis en profundad del surgimiento de cada uno de ellos y de los matices que los diferencian puede consultarse Uribe Mallarino (2012).

2 Entiendo los "discursos" como prácticas sociales (Van Dijk 1993; Fairclough 1998; Foucault 2002) que van más allá del habla o del lenguaje en tanto que sistema simbólico. Como tales representan, pero además "construyen" la realidad (Todorov 1991; Fairclough 1998; Potter 1998; 
En otras palabras, para estudiar cómo eran producidas la relaciones de alteridad hacia un grupo social determinado estimé oportuno observar el funcionamiento del discurso político al respecto, el diseño de políticas educativas dirigidas al colectivo y la implementación de las mismas en las escuelas. Para ello realicé ACD de las sesiones parlamentarias en las que se discutía sobre inmigración y educación durante los años 2000-2008, y trabajo de campo etnográfico durante un curso escolar (2004-2005) en administraciones públicas educativas y escuelas ${ }^{3}$.

A continuación, y después de haber esbozado brevemente la propuesta metodológica seguida, mostraré de forma somera el surgimiento y desarrollo de cada uno de los enfoques elegidos, la relación existente entre los mismos y la sinergia surgida entre ambos en mi investigación. Por último detallaré cómo fue la producción y análisis de los datos en la investigación, para finalizar con un apartado reflexivo entorno a la diversidad metodológica y la combinación de enfoques distintos en Ciencias Sociales.

\section{DIVERSIDAD METODOLÓGICA Y MULTI, INTER Y TRANS- DISCIPLINARIEDAD: ENCUENTROS Y DESENCUENTROS ENTRE ANÁLISIS CRÍTICO DE DISCURSO Y ETNOGRAFÍA}

Tanto el ADC como la Etnografía son enfoques metodológicos ampliamente practicados y teorizados en Ciencias Sociales. No obstante no es tan habitual conjugarlos en investigaciones concretas, y menos aún reflexionar a nivel epistemológico sobre las posibilidades que ofrece la combinación de ambos. En los apartados que siguen rescato las sinergias existentes entre los dos enfoques, que no una descripción exhaustiva de las características de cada uno de ellos, con el objetivo de visibilizar y mostrar al/la lector/a las "lagunas" en uno y en otro susceptibles de ser rellenadas en conjunción de ambos.

\subsection{Algunas notas sobre análisis crítico del discurso: de la de- construcción a la de-colonización de las prácticas discursivas}

Las diferencias entre el ACD y el Análisis de Discurso están claras, teniendo que ver con las temáticas abordadas (relacionadas con el estudio del poder, las ideologías, racismo, desigualdades sociales, etc. en el caso del ACD); y especialmente con la perspectiva -crítica-con la que ello se hace. Existe, por lo tanto, una intención política innegable en el ACD (Van Dijk 1999a, 2003).

\footnotetext{
Burr 2003).

${ }^{3}$ Todo ello me permitió contar con un material de 487 iniciativas parlamentarias (debates, preguntas orales y escritas, interpelaciones, proposiciones de ley, etc.), 117 entrevistas realizadas en centros educativos, 28 entrevistas realizadas en las administraciones públicas e innumerables relatos de observación y observación participante. Las entrevistas fueron realizadas por el equipo del Laboratorio de Estudios Interculturales, del que formo parte como investigadora.
} 
Hay, por otro lado, una diversidad tremenda en el seno del ACD. Para unos autores, como Fairclough (2001), el ACD es un método que debe enmarcarse en proyectos críticos de más alto alcance. Para otros, como Van Dijk, es algo más que una simple metodología, con dimensiones teóricas y aplicadas, que entraría dentro de lo que él denomina los Estudios Críticos del Discurso (Van Dijk 2008). En mi propuesta metodológica lo tomo como una forma crítica de acercarme al estudio de la realidad social, como una perspectiva y un conjunto de técnicas que sitúo en un marco metodológico más amplio -junto a otros enfoques y técnicas-, dentro del paradigma cualitativo.

El ACD supone una forma de posicionase dentro de las Ciencias Sociales que enfrenta diferentes formas de control social. Es una perspectiva de análisis que tendría su origen en paradigmas críticos de los años setenta como los Estudios Culturales, la Escuela de Frankfurt o la Lingüística Crítica. No obstante, no tomará forma hasta principios de la década de los años ochenta, estando influenciado e influenciando a diversas disciplinas. Contribuyeron a su desarrollo la Sociología, la Linguística, la Antropología, la Psicología Social, etc.

El principal objetivo del ACD es hacer que emerjan las intenciones existentes detrás de los textos, hablados y escritos. Ello va más allá de una simple interpretación de significados, dado que interpretar no implica necesariamente estudiar las consecuencias o fines de esos significados. En otras palabras, trata de desvelar las ideologías de los hablantes, trata de desvelar el rol que juegan los discursos en la pervivencia de las desigualdades y mantenimiento de jerarquías y mecanismos de dominación y lucha contra esa dominación:

(...) en los paradigmas más abstractos y formales, con frecuencia se descalifica y marginaliza a la investigación crítica tachándola de $<<$ política $>>$, y por tanto <<acientífica >>. El ACD rechaza tal evaluación (...) [y se esfuerza] por ser mejor que el análisis $<<$ ordinario $>>$ del discurso. Sus prácticas sociales y políticas no deberían contribuir solamente al cambio social en general, sino también a avances teóricos y analíticos (Van Dijk 2009: 151).

Para ello es necesaria la implicación del/la investigador/a en aquello que estudia (Martín Rojo et al. 1998), sin que esto suponga una "manipulación" de los datos para conseguir los resultados esperados (Wodak y Matouschek 1998). Siempre se ha de mantener una distancia crítica con respecto a los sujetos objeto de investigación.

Existe, en cierto modo, una analogía entre esto y la perspectiva etic/ emic utilizada en la Etnografía: ese equilibrio buscado -aunque no siempre conseguido- entre el análisis e interpretación de la realidad social por parte del/a investigador/a y la visión de los hechos sociales que tienen los propios sujetos investigados, como dos perspectivas imprescindibles y complementarias en la interpretación de las culturas. Y es precisamente aquí donde se han hecho críticas de manera frecuente a la Etnografía, por presentar en ocasiones análisis 
“excesivamente simplistas” y que podrían ser mejorados con el $\mathrm{ACD}^{4}$ :

El tratamiento del discurso en Antropología es bastante peculiar [ya que] nuestra masiva utilización de los discursos, como consecuencia del recurso a materiales provenientes de la información, ha llenado las monografías de discursos tratados con cierta simplicidad (García García 1996: 11).

Simplismo que sería el resultado de: 1). Dar más peso al enfoque emic, otorgándole más protagonismo del necesario, por encima incluso de las interpretaciones y análisis del/a propio/a etnógrafo/a; 2). Confundir emic con etic; e incluso 3.) La adopción por parte del/a etnógrafo/a categorías emic como propias de manera inconsciente ${ }^{5}$.

Pero ¿por qué fue tan oportuno realizar ACD dadas las características y los objetivos de mi investigación? En primer lugar he de destacar que fue determinante el tipo de discursos que me proponía estudiar: discursos de élite (Van Dijk 1993, 2003), procedentes de parlamentarios, de cargos políticos y técnicos de las administraciones educativas y de profesorado, con "poder persuasivo en la construcción del consenso y la hegemonía ideológica (...)" (Van Dijk 1997: 16). Los discursos de élite vehiculan las creencias y actitudes de los "grupos dominantes", que lo son porque tienen un acceso privilegiado a los medios de comunicación y al discurso público: mayor visibilidad, influencia, difusión, persuasión e imposición; mayor capacidad de crear opinión pública -en definitiva- porque son discursos autorizados que proceden de fuentes autorizadas (Ibídem). Son discursos vertebrados y vertebradores de relaciones de poder y jerarquías. En segundo lugar, pero muy relacionado con lo anterior, en la elección del diseño metodológico influyó que mis puntos de partida a nivel teórico fueran los trabajos sobre la construcción social de la realidad de P. Berger y T. Luckmann (1967), y dentro de este macro posicionamiento teórico los postulados sobre la construcción social de la diferencia (García Castaño et al. 1999). La de-construcción en sí misma, por lo tanto, era un objetivo de mi trabajo: de-construir los imaginarios sociales sobre "la inmigración como problema -y la imagen del alumnado inmigrado de nacionalidad extranjera

4 Es la dificultad de implementación de la metodología con respecto al análisis de los datos producidos lo que supone una debilidad en sí misma. Si ello se puede suplir con herramientas que han sido más desarrolladas desde otras perspectivas, nos permitirá ser más precisos y concretos. Dichas carencias en el análisis que a menudo encontramos en el quehacer etnográfico -debido a la complejidad misma de los datos producidos- pueden abordarse con técnicas del ACD, procedentes en su mayor parte de la Linguística, y que permiten conocer la intencionalidad de los hablantes a través de un estudio detallado de cuestiones como: la persona usada por el/la hablante para esbozar ideas, las figuras estilísticas empleadas, la recurrencia y/u desatención a de/a determinados temas, etc., que propician un análisis más profundo de los discursos emic.

5 González Echevarría (2009) nos aporta un razonamiento muy sugerente al respecto al señalar que la dicotomía etic/emic es errónea desde el punto de vista gnoseológico y metodológico, apuntando a la existencia diferenciada de ambos enfoques al tiempo que la imposibilidad de disociarlos en el análisis. Ello, entiendo, redunda en la tesis sostenida por García García (2009) sobre el tratamiento "simplista" dado frecuentemente en Antropología a los discursos. 
como "problemático"-, para lo que el ACD me aportaba claves fundamentales ${ }^{6}$. Este enfoque de análisis permitía desvelar los procesos de construcción de relaciones sociales, de acontecimientos concretos, de los propios sujetos (alteridad/identidad), pero al mismo tiempo analizar las implicaciones de esas construcciones socio-culturales (Martín Rojo et al. 1998): diferenciación, exclusión, marginación, estigmatización, etc. La idea principal que guía todo este posicionamiento es la de que los discursos -en tanto que prácticas sociales con poder per-formativo- juegan un papel esencial en la construcción social de la realidad, y por ende, en la construcción de la diferencia.

En cuanto a las principales críticas que se han realizado al ACD, he de destacar su excesivo énfasis en el estudio del poder político y la ideología, lo cual termina haciéndolo reduccionista (Fairclough 1998). Existen otros poderes no "estrictamente políticos", sino más bien "institucionales" en sentido más amplio (léase para mi investigación las administraciones educativas, la escuela como institución y el cuerpo de técnicos y funcionarios que trabajan en ellas), que estimo muy pertinente analizar desde el ACD. Estos otros discursos también de élite- construyen asimismo la realidad social y contribuyen a la generación de representaciones y a la construcción social de problemáticas. En definitiva, habría que tener en cuenta que el discurso de élite excede al discurso político (Van Dijk 2003). Por otra parte, y de nuevo coincidiendo con Fairclough (1998), sería necesario un posicionamiento claro dentro del ACD sobre cómo relacionarse con otras metodologías y enfoques de análisis de la realidad social, como la Etnografía, dado que en función de la disciplina desde la que trabaje el/ la investigador/a, vamos a encontrar cuestiones que se confunden y presupuestos que se solapan.

Ello acontece precisamente con el trabajo etnográfico: tanto uno como otro estudian imaginarios colectivos y representaciones sociales a través de discursos y prácticas discursivas, enfatizando en dicho proceso el papel del contexto donde se insertan estas. Mi trabajo de investigación terminó situándose a ambos lados, y mi propuesta es la combinación de los dos enfoques porque estimo que son complementarios y en mi caso en concreto, cada uno de ellos arrojó luz sobre cuestiones importantes de mi objeto teórico de estudio -las relaciones de alteridad-, que pienso puede ser extrapolable a otras investigaciones sociales.

\subsection{Algunas notas sobre Etnografía: de Malinowski a la etnografía virtual}

Cuando hablamos de Etnografía (aunque tendríamos que decir más bien etnografías) estamos aludiendo a un proceso metodológico dentro de la

${ }^{6}$ Posteriormente advertí que también era necesaria una labor de de-colonización de ciertas categorías socio-culturales que fueron centrales en mi trabajo (a saber, "raza"/etnia, clase y género, entre otras), por lo que el acercamiento entre el ACD (Fairclough 1998; Van Dijk 2008) y la teoría decolonial (Walsh 2007; Grosfoguel 2012) está siendo tomado en consideración en mis trabajos actuales. 
perspectiva cualitativa. Se trata de algo más global que el ACD, porque también se ocupa de la recogida/producción de datos y no solo del análisis. La variedad etnográfica es muy importante (Werner y Shoepfle 1993; Velasco y Díaz de Rada 1999; Hammersley y Atkinson 2001), por lo que existen muchas definiciones, y estas no siempre son concordantes. Además actualmente estamos ante una diversificación de las formas de hacer Etnografía: etnografía virtual (Hine 2004), etnografía multi-sutiada (Marcus 1995, 2008), etnografía colaborativa (Lassiter 2005), etnografía reflexiva (Dietz y Mateos 2010; Dietz 2012), lo que para posicionamientos más tradicionales supone aún un desafío difícil de encajar y aceptar.

Para hablar de las características de la Etnografía que son ampliamente aceptadas y sobre las que suele haber acuerdo dentro de la comunidad científica tenemos que remontarnos al trabajo de B. Malinowski Los argonautas de Pacífico Occidental (1922). Allí el autor definía los presupuestos básicos de la que sería conocida como la etnografía científica (Gómez 1995), que ponía en valor y reivindicaba la participación del etnógrafo en las realidades estudiadas:

Dentro de este entramado hay que insertar los imponderables de la vida real y el tipo de comportamiento. Estos datos se consiguen gracias a la observación minuciosa y detallada (...) posible a partir de un estrecho contacto con la vida indígena. (...) La meta es, en resumen, llegar a captar el punto de vista del indígena, su posición ante la vida, comprender su visión de su mundo. (Malinowski 1993: 111).

Una segunda característica, ampliamente aceptada, de la metodología etnográfica tiene que ver con su capacidad flexible y adaptativa:

Cuantos más problemas se plantee sobre la marcha [el etnógrafo], cuanto más se acostumbre a amoldar sus teorías a los hechos y a ver los datos como capaces de configurar una teoría, mejor equipado estará para su trabajo. Las ideas preconcebidas son perniciosas en todo trabajo científico, pero las conjeturas son el don principal de un pensador científico (...) (Ibídem: 96).

La pretensión de holismo sería otro de sus rasgos definitorios:

La aproximación holística es considerada universalmente por los antropólogos como la característica distintiva de la etnografía clásica. (...) la noción de etnografía holística recuerda de inmediato a los clásicos. Antropólogos como Franz Boas (indios Kwakiutl, 1909), Bronislaw Malinowski (nativos de las islas Trobriand, 1922), Robert Lowie (indios Crow, 1935), así como otros que siguieron sus pasos, encajan en esta categoría (Werner y Schoepfle 1993: 121).

Buscando con ello recoger con rigor y seriedad la totalidad de aspectos y fenómenos sociales de la realidad estudiada.

Y debemos hablar también de la actitud de relativismo cultural de la Etnografía, que nos ayuda a evitar el etnocentrismo en los casos en los que 
los hechos sociales estudiados nos resulten distantes y poco familiares a los códigos culturales propios; al igual que la actitud de extrañamiento, que propicia la distancia crítica necesaria en los casos de una mayor familiaridad con las realidades estudiadas.

Hay acuerdo también sobre que realizar trabajo etnográfico va más allá de la simple utilización de unas técnicas de recogida/producción de información determinadas (observación participante y entrevistas, principalmente):

Las técnicas de campo en y por sí mismas no pueden componer una etnografía. Podría concebirse que un investigador usase una técnica de campo fundamental (por ejemplo, la observación participante o la entrevista), muchas técnicas a un tiempo (...), y que sin embargo no llegase a hacer un estudio etnográfico. Es cierto, se tendría el material con el que se construye la etnografía, pero eso no sería para vanagloriarse demasiado, ya que la etnografía está hecha de material cotidiano (Wolcott 2005: 128).

El uso de dichas técnicas no sería suficiente, sino requerimiento indispensable.

Por último señalar a la Etnografía como una propuesta de descripción, y lo que es más importante, de interpretación cultural (Geertz 1973; Werner y Schoepfle 1993; Wilcox 2005; Wolcott 2005). En este punto encontramos de nuevo sinergia con el ACD: ambos tratan de desvelar los significados detrás de la realidad socio-cultural que se estudia, aunque ello supone algo central en Etnografía y solo un rasgo más en $\mathrm{ACD}$. Este último se muestra más interesado en ejercitar una orientación crítica para conocer como "el discurso participa de la reproducción del abuso de poder (la dominación) (...), sus consecuencias sociales (...) [y] la propia lucha contra esa dominación" (Van Dijk 2010: 179), lo que no es imperativo sino facultativo en Etnografía.

En cuanto a las características de la Etnografía sobre las que existe mayor desacuerdo entre quienes la practican estarían, por un lado 1). La disciplina en la que ubicarla; por otro 2). La discusión sobre qué culturas son las que interpreta/ estudia; y 3). La implicación política de la misma.

El debate sobre la adscripción de la Etnografía a unas u otras disciplinas no es más, bajo mi punto de vista, que el producto de unas dinámicas encaminadas a copar mayores espacios de poder dentro de la academia. La Antropología ha afirmado la misma como "su seña de identidad", entendiendo que "el término etnografía alude al proceso metodológico global que caracteriza a la Antropología Social, extendido luego al ámbito general de las ciencias sociales" (Velasco y Díaz de Rada 1999: 18). Es cierto que desde la Antropología se ha teorizado en mayor medida sobre Etnografía; y son los/as antropólogos/as quienes más etnografías han producido hasta el momento, desde que Malinowski sentara las bases con su trabajo con los trobiandeses. No obstante, desde la Sociología, por ejemplo, se aboga más por una defensa de la Etnografía en tanto que metodología de las Ciencias Sociales en su conjunto.

Con respecto al segundo debate -la Etnografía como un proceso de interpretación de qué culturas- en cierto modo fue una discusión más en boga 
en el pasado, cuando se entendía que la Antropología era "la ciencia del otro cultural" y la Sociología "la ciencia del nosotros". Evidentemente hay un sesgo occidentalocéntrico tremendo en esta separación. A día de hoy está bastante extendido realizar Etnografía at home, pero es que además no es posible -ni deseable- definir de manera categórica quienes son "los otros" y quienes "el nosotros": dependerá de quien realice la categorización -y ello de cómo se establezcan las relaciones de poder, en caso de que las haya-. El relativismo cultural y el extrañamiento serán actitudes que vertebren un tipo y otro de hacer Etnografía, pero ambas posibilidades son igualmente practicadas -y simultaneadas- actualmente. En mi investigación entendí que la realización de Etnografía era un ejercicio para el análisis y la interpretación tanto de la alteridad como de la mismidad, del "otro cultural" y del "nosotros" (categorías ambas construidas constantemente, procesuales per se, y que en ocasiones no presentan fronteras claras). Es por ello que no podía limitarme a conocer las representaciones e imaginarios sociales sobre los/as inmigrantes y los/as alumnos/as inmigrados/a de nacionalidad extranjera en España sin con ello acercarme inevitablemente con mis análisis al estudio de la construcción de la identidad (los "nacionales", los "españoles", el "alumnado autóctono"). En mi caso, tanto una actitud de extrañamiento como una posición de relativismo cultural fueron necesarios a lo largo de todo el trabajo de campo, dado que trataba de etnografiar prácticas y discursos en una institución que me era tremendamente familiar, donde crecí y me socialicé, y de la que siempre había formado parte: la escuela española; pero dotada de características nuevas: una creciente diversidad cultural procedente de la llegada a las aulas de alumnado inmigrado de nacionalidad extranjera ${ }^{7}$.

Un último debate abierto en la actualidad, y que es interesante destacar, tiene que ver con el compromiso político dentro de la Etnografía. Los posicionamientos explícitamente comprometidos son relativamente recientes en la historia de esta metodología (Lather 1986, cit. en Hammersley y Atkinson 2001). Para muchos es objetivo prioritario en la investigación producir conocimiento (Ibídem), sin otorgar demasiada importancia a la intervención social y/o la militancia. Incluso encontramos reacciones contrarias a ello, exigiendo la no implicación para curarnos de los riesgos de "convertirnos en nativos". No obstante cada vez son más frecuentes los trabajos etnográficos realizados desde posicionamientos postestructuralistas, postmodernos, neo y postmarxistas y decoloniales (Haraway 1991; Scheper-Hughes 1995; Guber 2010; Dietz y Mateos 2010) que reivindican un compromiso político dentro de la Etnografía. De nuevo habría en ello posibilidad de un punto de encuentro entre el ACD y la Etnografía.

${ }^{7}$ La llegada de alumnado de nacionalidad extranjera en España se produce a partir de los años noventa del pasado siglo, no siendo notable estadísticamente su presencia en las aulas hasta bien avanzada dicha década. Así, en el año 1995 las estadísticas escolares señalaban un 0.7\% de alumnado extranjero, en el año 2000 ya eran un $2 \%$ y a finales de la primera década del presente siglo suponían el $9.5 \%$, dato muy similar a la media actual. No obstante la distribución del dicho alumnado no ha sido uniforme en todo el territorio (ver Capellán de Toro et. al. 2013). 


\section{APUNTES PARA UNA PROPUESTA METODOLÓGICA: HACIA UNA "ETNOGRAFÍA DE LOS DISCURSOS}

A continuación apuntaré las razones que me han movido para estimar oportuna la combinación de ACD y Etnografía en mi investigación, y describiré el procedimiento seguido para ello.

Para una interpretación lo más holista posible de cualquier discurso es preciso recurrir a la observación, igual que hacemos con otras prácticas cuando son objeto de investigación:

En la vida real no existe lo observable y su descripción, como si se tratase de dos realidades socialmente distintas. A no ser, obviamente, que se admita - cosa que estaría dispuesto a asumir-, que entre los fenómenos que deben ser observados están los propios discursos. Incluso la descripción discursiva de acontecimientos, aunque no se pueda verificar referencialmente, es una conducta social que puede resultar más trascendente que aquello que desfiguradamente relata (García García 2000: 78).

Un simple análisis de contenido nos ofrecería una descripción poco profunda, simple y lineal de aquello que estamos estudiando. Para evitarlo podemos interrogar e interpretar los discursos. Es decir, podemos "etnografiarlos": la puesta en práctica del ACD en el curso de una investigación etnográfica, estaría aportando una mayor profundidad analítica porque nos permite observar más allá de los datos producidos a través de la descripción. Uno de los objetivos en este quehacer, como apuntaba más arriba, es evitar y/o deshacer el simplismo en la utilización de los discursos emic que en algunos casos incurrimos cuando realizamos Etnografía (Ibídem); y llegar a lo que en teoría plantea conseguir la Etnografía, y cualquier análisis de discurso, a saber, obtener respuestas acerca de los procesos de construcción, la intencionalidad y consecuencias de los discursos:

Todo análisis de discurso admite, desde el punto de vista de la interlocución, una respuesta consistente a cuestiones de este tipo: ¿por qué me cuenta usted a mí esto?, ¿por qué me lo cuenta así?, ¿a quién no contaría usted esto que me está contando a mí?, ¿cómo contaría usted esto mismo a otra persona?... Las respuestas, directas o indirectas, a cuestiones de este tipo forman parte importante del análisis de discurso (Ibídem: 81).

En otras palabras, localizar "estrategias discursivas" distintas formas de comunicar. Como comentaba anteriormente, algunas

${ }^{8}$ Una "estrategia discursiva" es una forma concreta de construir argumentos para legitimar y respaldar determinados discursos. Es una manera de persuadir al oyente (Charaudeau y Maingueneau 2005), que puede servir para establecer diferencias que terminen generando desigualdades. Una "estrategia discursiva" trata de esgrimir argumentos para crear consentimiento y convencer a la audiencia, modificando la realidad para que sea interpretada de una determinada manera y no de otra, y no dejando opción a alternativas. 
etnografías, a pesar de trabajar tanto con discursos carecen de un tratamiento analítico profundo de estos, dada la complejidad de los mismos y, principalmente, porque prestan más importancia y dan mayor validez a las palabras de los informantes, subestimando con ello las propias observaciones (interpretaciones). Por su parte, algunos trabajos dentro del campo del Análisis del Discurso y del ACD -sobre todo aquellos de corte más linguíístico- terminan realizando análisis demasiado ligados al "lenguaje". Trabajar de manera combinada con ACD y Etnografía podría ayudar a superar ambos escollos, saliendo al campo y zambulléndose en el mismo para conocerlo de manera que se obtenga un conocimiento adecuado del contexto de producción de los discursos, como primera salvaguarda para una interpretación holista de la realidad social.

Por todo ello en mi investigación he querido caminar hacia lo que llamo una "etnografía de los discursos", para abordar mi objeto teórico de estudio -la construcción de relaciones de alteridad- a través de:

1. La de-construcción de los discursos estudiados, cuestionándome para ello sobre sus lógicas de funcionamiento.

2. El estudio de los contextos de producción y de emisión de dichos discursos.

3. $\quad \mathrm{Y}$ el contraste permanente entre las perspectivas emic y etic: la comparación constante entre los discursos emitidos por los/as sujetos observados y los producidos por mi misma como investigadora; la puesta en diálogo continua entre las descripciones de los/as informantes y mis interpretaciones de lo directamente observado. detallado.

Mostraré a continuación dicha propuesta metodológica de un modo más

\subsection{Funcionamiento de categorías socio-culturales según el contexto}

Situándome como lo he hecho en un marco que bebe de distintos enfoques metodológicos y disciplinas no podía sino aproximarme a mi objeto de estudio desde una perspectiva de-construccionista que cuestione los esencialismos (Foucault 1970; Derrida 1975; Berger y Luckman 2006). De igual modo, en este proceso, preguntarnos por cómo ciertas categorías han sido producidas desde una óptica occidentalocéntrica por la colonialidad del poder/saber (Quijano 2000; Grosfoguel 2006, 2011)9 ayudará en esa labor de de-construcción.

Para ello es necesario "problematizar" todo aquello establecido como la

9 La colonialidad del poder refiere que "la raza", como criterio de clasificación social de la población a nivel mundial, que opera desde la época de dominio colonial, está en la base y supone un eje fundamental del actual patrón de poder (Quijano 2000). La colonialidad del saber, como una de las partes de la colonialidad del poder, hace referencia a la violencia epistémica -y epistemicidio- que entraña el eurocentrismo tomado como única manera valida de "conocer" (Grosfoguel 2006, 2011). 
normalidad, realizando un ejercicio crítico para cuestionar incluso nuestra forma de acercarnos a las realidades sociales que estudiamos. ¿Por qué en la actualidad se construye como legítimo cerrar las fronteras nacionales a "cal y canto"? ¿por qué el "inmigrante extranjero" es construido como una figura de alteridad total y como el "enemigo"? ¿por qué las escuelas occidentales y occidentalizadas entienden que (determinados/as) alumnos/as inmigrantes extranjeros/as perturban y deterioran el normal curso de las actividades educativas? Estos interrogantes, que eran tomados como afirmaciones y verdades dadas en gran parte de los discursos públicos analizados, fueron mi punto de partida para analizar los procesos de construcción de alteridad. En otras palabras, de-construir los discursos supuso en primer lugar preguntarme por sus lógicas de funcionamiento. Para ello fue indispensable una labor profunda y constante de contextualización de los mismos.

Los contextos funcionan como moduladores de los discursos en tanto que conjuntos de relaciones que regulan el orden de los mismos a través de estrategias, facilitado con ello la emergencia de algunos enunciados y sub-dimensionando otros, en el proceso de caracterización y definición de realidades sociales (Foucault 1970). Es por esto que los contextos en los que se produce el habla imprimen significado y establecen el alcance de las manifestaciones verbales y las posibles interpretaciones que se puedan realizar al respecto (Vasilachis 1997). Un mismo discurso emitido en contextos diferentes tendrá, por lo tanto, significaciones distintas porque "hablar es una conducta condicionada por los interlocutores, negociada implícitamente en su desarrollo y expuesta al peligro de no ser entendida fuera de la situación comunicativa. Es el resultado de un conjunto de estrategias que afectan no sólo a los aspectos formales del discurso, sino también a sus contenidos" (García García 2000: 85). Siendo así, parto del supuesto de que no emergerán las mismas interpretaciones de un discurso que entienda la inmigración como problema en el transcurso de un debate parlamentario y del mismo discurso emitido en una reunión de una asociación de padres y madres de alumnos/as de un colegio con presencia de alumnado inmigrante. Pero tampoco ambos discursos tendrán el mismo alcance y repercusión social. Es por ello necesario "situar los discursos en sus contextos cognitivos, sociales, políticos, históricos y culturales para entenderlos" (Van Dijk 1999b: 252), para poder analizar la diversidad de significados e implicaciones sociales de una misma afirmación. "Las frases o las palabras no pertenecen a un determinado discurso en particular, de hecho el significado de lo que decimos depende más del contexto discursivo, del marco conceptual general en el que nuestras palabras están incrustadas" (Hall 2001, cit. en Burr 2003: $\left.66^{10}\right)$

La propuesta, por lo tanto, sería considerar una serie de dimensiones contextuales que propicien conseguir interpretaciones de carácter más holístico. Dichas dimensiones tendrían que ver y estarían contenidas en las coordenadas

\footnotetext{
${ }_{10}$ Traducción propia del original.
} 
de tiempo ${ }^{11}$ y espacio ${ }^{12}$. En este sentido contextualizar un discurso supondría, por ejemplo, analizar un texto escrito (el articulado de una ley, de una política social, etc.), realizar entrevistas a quienes se encargaron del diseño y redacción del mismo (parlamentarios, funcionarios de la administración, etc.) y realizar entrevistas también a quienes afecta directamente: a quienes implementan dichas leyes y/o políticas y a quienes son objeto de intervención a través de las mismas (profesorado, alumnado, etc.). Todo ello en un marco general de observación y observación participante en escuelas, en el Parlamento, en las sedes de la administración pública educativa. Veamos un ejemplo de ello. A continuación cito dos fragmentos de entrevistas realizadas a un profesor y a una orientadora educativa de dos centros escolares distintos. En ambas se trata el tema de racismo en la escuela:

\section{Ejemplo 1}

(...) No. Racismo como lo entendemos no hay. Es decir, siempre está el típico insulto de "moro de mierda", para que nos entendamos. Pero en el sentido de que los niños siempre intentan tirar donde más duele. "Ah, pues tú eres un racista.” ¿qué te ha dicho? Pues simplemente moro. ¿Moro? Pues sí, eres moro. A mí me puedes decir lo que tú quieras... blanco, pues blanco, si fuera chino me dirías chino, pero no racismo en el sentido de que no se integren en la clase, de que se les mantenga aparte. (Entrevista a profesor de un centro de la provincia de Almería).

\section{Ejemplo 2}

Claro, pero eso es como algo cultural... es que decir "colombiano" no es ofensivo, muchas veces dicen "el moro ese" para ofender: es como decir "mira que feo eres", "mira que tonto eres" o "eres un moro". (Entrevista a orientadora educativa de un centro de la provincia de Sevilla).

En ambos casos, al hablar de racismo, emerge una misma categoría: "moro"; pero entendida de manera radicalmente diferente - por lo que su uso, implica relaciones sociales igualmente diferentes- por cada una de las personas entrevistadas. Para poder interpretar y explicar por qué sucede esto, vamos a situar dichos discursos en sus ámbitos espacio-temporales, y vamos a considerar para ello los contextos cognitivos, políticos, históricos y culturales anteriormente mencionados.

Con respecto al contexto cognitivo, entendido como el conocimiento del

11 En la contextualización temporal se atendió al momento en el que se produjo el discurso analizado: en qué año y en qué legislatura; para interpretar dichos discursos en función de qué partido político estaba en el Gobierno en ese momento, cual era la legislación de extranjería vigente, qué políticas sociales operaban, etc.

${ }^{12}$ La contextualización espacial fue entendida como las características del lugar donde se produjo la interacción comunicativa; considerando para la interpretación de los discursos si los mismos fueron emitidos en el Parlamento, fueron obtenidos sobre la base del diálogo en la realización de una entrevista en un despacho, en un aula, etc. o se recogieron a partir de un encuentro informal con profesorado en el patio de un colegio, por ejemplo. Es decir, importaba tanto la dimensión física del espacio como las condiciones de producción de los discursos en el mismo. 
mundo que se posee y se comparte, tendríamos que preguntarnos ¿qué significa la categoría "moro" para las personas entrevistadas? Para el profesor se trata de una categoría equivalente a la de "blanco". Sin embargo, para la orientadora significa "mira qué feo eres". En otra entrevista realizada durante mi trabajo de campo un chico marroquí afirmaba lo siguiente:

Yo tengo amigos buenos y amigos malos, y los amigos malos me molestan. Los malos a mí no me llaman por mi nombre, Mohamed, me dicen "moro" todo el día, y ya está. Y yo les digo: i ¡ime llamo Mohamed!!!; yo les digo eso muchas veces y nadie me hace caso. En mi clase hay gente buena, pero hay otros que son... (Mohamed, 14 años).

Con todo ello podríamos interpretar que cuando el profesor afirma "no hay racismo", y al mismo tiempo "¿Moro? Pues sí, eres moro. A mí me puedes decir lo que tú quieras... blanco, pues blanco", está aconteciendo un proceso de esencialización de categorías socio-culturales, donde son obviadas las relaciones de poder que producen y significan las mismas.

Con respecto al contexto social en que son producidos los discursos, es importante conocer que la inmigración es un fenómeno altamente problematizado $^{13}$, que se entiende que la mayor parte de los inmigrantes son marroquíes ${ }^{14}$ y que son precisamente las personas de esta nacionalidad a quienes se identifica con la categoría "moro". Por lo tanto, tendríamos un elemento más para entender que "moro" no es un marcador de carácter positivo o neutro. Véase este otro relato de un profesor que se muestra indignado por las atribuciones de "terrorismo" realizadas hacia un alumno por el propio director del centro donde trabaja:

El año pasado (...) yo denuncié un acto de racismo clarísimo porque no querían matricular a un niño marroquí en el centro porque el centro está muy bien considerado y el señor director dentro de su trono pues intentó por todos los medios convencerme de que el niño que no podía entrar porque él tenía alumnos que estando al lado no habían podido entrar y por qué un "moro" va a entrar y luego poner una bomba y dije yo: "hasta aquí hemos llegado... (Entrevista a profesor de un centro de la provincia de Córdoba).

${ }_{13}$ Para dar contexto en este sentido a los discursos analizados en mi investigación, traté de conocer en profundidad la evolución del tratamiento político de las migraciones internacionales -y su gestión en el ámbito de lo educativo- a varios niveles: en el marco de la Unión Europa, en el conjunto de España y a nivel regional; dado que las tres dimensiones están relacionadas e influenciadas en materia de gestión de los flujos migratorios y políticas de integración social (de las que forman parte las políticas educativas).

${ }_{14}$ Es curioso en este sentido que el mayor porcentaje de extranjeros en España sea de personas de alguna nacionalidad europea, pero en el imaginario colectivo prima la imagen del inmigrante marroquí frente a éstos, que son percibidos en muchas ocasiones como turistas, a pesar de residir habitualmente en el país. 
El contexto político donde se insertan los discursos analizados, por su parte, se caracteriza por la proliferación de legislaciones represivas hacia la entrada y presencia de población inmigrante extranjera en el país, lo cual viene a reforzar más aún la imagen de la inmigración como problema. Así lo mostraba un político en sede parlamentaria:

[tenemos] el mayor despropósito en política de inmigración en Europa, criticado por todos los países de la Unión Europea, y que está provocando que haya alrededor de... inmigrantes, muchísimos inmigrantes irregulares, que están en nuestra Comunidad y en nuestro país a los que tenemos que hacerles frente (Grupo Parlamentario Popular, Parlamento de Andalucía).

El contexto histórico también nos aclara bastante en este sentido, dado que el pasado colonial de España con respecto a Marruecos puede explicar en parte la construcción de la categoría "moro" y su funcionamiento en la actualidad, que hereda significados y tópicos prejuiciosos de esa época y momentos posteriores que perviven a día de hoy. Véase el siguiente ejemplo, extraído de un grupo de discusión realizado con padres y madres de un centro escolar:

No viene al caso pero te lo voy a contar, una prima de mi marido a raíz de que vivían unos cuantos "moros" al lado de su casa, nada más que de pasar por la puerta cogió tuberculosis y si vienen familias y demás pues que vengan con sus vacunas puestas y con sus cosas porque no hay derecho que en el s. XXI cojamos enfermedades como cogió la prima de mi marido que es del s. XVI, y ésta mujer se escapó porque no era su día, pero que estuvo en coma y todo a raíz. de los "moros". (...). Es que ellos ni tienen higiene (...) es que esas enfermedades hacía años que no se veían, desde la guerra como dicen (Grupo de discusión con padres y madres de un AMPA de la provincia de Huelva).

Por último, y con respecto a la última dimensión contextual considerada, la cultural, el análisis se complejiza, por las propias dimensiones del concepto, y sin duda es posible incurrir en reduccionismos al respecto. Pero tomemos por un momento -y con fines estrictamente analíticos- una idea de cultura como algo que tiene que ver con religión y con lengua (obviamente, insisto, con este ejercicio limitamos enormemente los procesos que la conforman). En este caso de nuevo emergen características del momento actual que nos desvelan significados existentes debajo de la categoría "moro". El Islam y el árabe son marcadores tremendamente estigmatizados en los años en los que se llevó a cabo la investigación (lo siguen siendo en la actualidad). A ello contribuyó enormemente la sucesión de atentados terroristas perpetrados por fundamentalistas musulmanes en territorios en Nueva York, Londres y Madrid. Y ello, sin duda, significa de manera explícita - de nuevo- la categoría "moro" y determina unas relaciones sociales con quienes son categorizados como tales. Así lo reconocía una maestra entrevistada:

(...) mientras que vengan como vienen, a hacer trabajos que la gente de 
aquí no quiere pues yo tampoco entiendo por qué hay tanta furia contenida en esto porque no vienen a quitar nada que tú no quieras (...) pero claro luego después hay más matizaciones, están las matizaciones ahora un poco de los atentados, ahora como tenemos muy cercana la inmigración árabe pues los fundamentalismos árabes y eso que son un poco incompatibles con la cultura que tenemos aquí, con la cultura nuestra que llevamos, además se tiene esa historia ahí de terrorismo islámico y claro les viene fatal a los demás pobres que no tienen culpa de nada (Entrevista a una maestra de un colegio de la provincia de Huelva).

La pregunta que resta después de estos apuntes, y con ello vuelvo a la idea con que iniciaba este apartado, sería: ¿podría tener otros significados la categoría "moro", y los discursos articulados alrededor de misma, si varían las coordenadas contextuales espacio-temporales? O dicho de otra manera ¿los contextos cognitivos, sociales, políticos, históricos y culturales explican el funcionamiento de las categorías socio-culturales? La respuesta ya ha sido esbozada. Pero quizá lo más importante es seguir insistiendo en que con una contextualización de los discursos definida en estos términos podemos conocer y tener una mayor comprensión de las relaciones existentes entre prácticas lingüísticas y fenómenos extra -o no- lingüísticos (Wodak y Matouschek 1998). Nos sirve por lo tanto para conocer acerca de si lo que se dice está relacionado con las dimensiones espacio-temporales en la que se dice; si dichas dimensiones influyen también en cómo se dicen las cosas; y para saber también si dichos discursos (que tienen un determinado contenido y una determinada forma) determinan o influyen de alguna manera en la emergencia de unas prácticas y no otras (ver Olmos Alcaraz 2009, 2010, 2012; Olmos Alcaraz y Rubio Gómez 2013).

Por ejemplo, se trataría de estudiar si el discurso de los políticos (que en el contexto estudiado, y sobre la materia analizada en cuestión -las migracioneses "políticamente correcto") presenta las características que presenta por el contexto espacial en el que se emite (el Parlamento, un espacio muy formal, público y al que tienen acceso medios de comunicación y la ciudadanía y en el que es posible la "sanción social"); o, por ejemplo, también analizar si un posible cambio de opinión un partido político en concreto se debe a un cambio de dirección del mismo en materia de política migratoria o, por el contrario, tiene más que ver con una estrategia política de oposición producto de un cambio de signo del partido en el Gobierno. Al hacer esto, en el caso concreto de mi investigación, dejo claro que estoy trabajando un determinado tipo de discursos -discursos de élite (Van Dijk 1997) - dotados de una capacidad especial de determinación de los hechos sociales, con más poder que otros discursos para crear la realidad social: para conservar el status quo o para iniciar cambios sociales.

En la labor de de-construcción es fundamental trabajar, por otro lado, otorgando el mismo valor a las perspectivas emic y etic. El contraste entre los discursos de los informantes y mis observaciones e interpretaciones de dichos discursos ha sido continuo en el proceso de investigación, suponiendo una de 
las estrategias metodológicas centrales de cara a conseguir un análisis lo más holista posible contribuyendo a la de-construcción de las lógicas de producción de alteridad. Por ejemplo, en el curso de mis entrevistas a profesorado encontré en muchas ocasiones que al preguntar "cómo era la escolarización de la población inmigrante extrajera”, una de las respuestas más frecuentes fue que "no había discriminación hacia ellos" (emic) porque "todos los niños/as son tratados como iguales" (emic). No obstante al realizar observación participante en las aulas comprobé que el alumnado inmigrante extranjero que no tenía un determinado nivel de conocimiento de español (variable en función del colegio) no participaba de igual manera que sus compañeros en las clases (etic). Es decir, si bien para el profesorado esas dinámicas no son consideradas discriminatorias, mi análisis era otro. Las perspectivas emic y etic proporcionan por lo tanto distintos puntos de vista de la realidad social, propician poder contar con información que es complementaria, facilitan una compresión más holista de los hechos observados. Todo ello es lo que incrementa la significatividad del análisis. En el ejemplo narrado podemos ver que la noción de discriminación es construida de manera distinta desde una perspectiva emic y desde una perspectiva etic: para los/as docentes no existe discriminación porque incurren en un proceso de naturalización de las diferencias, construyendo la igualdad solo con respecto al alumnado autóctono y no sobre la base de la diversidad que aporta la llegada de población inmigrante extranjera. Es por ello que excluir a un/a niño/a -inmigrante extranjero/a- del seguimiento normalizado de las clases no se considera discriminación. Sin embargo con un análisis en profundidad de los discursos observados, lo que vemos son lógicas de construcción de alteridad funcionando en los espacios educativos, imperceptibles si consideramos solo la perspectiva emic y igualmente imperceptibles si nos quedamos sólo con la perspectiva etic.

Para concluir con este apartado, y resumiendo la propuesta realizada, mi investigación trató de unir algunas de las ventajas que caracterizan al ACD y con algunos de los logros de la Etnografía. En este quehacer la de-construcción de los discursos ha sido parte esencial de todo el proceso investigativo, contextualizando para ello espacio-temporalmente los discursos analizados y estableciendo un diálogo continuo entre las perspectivas emic y etic.

\subsection{Sobre el proceso de análisis: ámbitos y plantillas}

Los discursos que fueron analizados en mi investigación se corresponden con cuatro ámbitos de trabajo. Lo que he llamado ámbito político, ámbito técnico-administrativo, ámbito normativo y ámbito escolar. Este paso fue dado para imprimir "orden” a nivel analítico en la realidad a estudiar, aunque siendo consciente en todo momento de los reduccionismos a que ello me enfrentaba. En cada uno de estos ámbitos encontré distintos tipos de discursos, que paso a describir a continuación.

Lo que he denominado ámbito político se componía a su vez de discursos

EMPIRIA. Revista de Metodología de Ciencias Sociales. N. ${ }^{\circ}$ 32, septiembre-diciembre, 2015, pp. 103-128. ISSN: 1139-5737, DOI/empiria. 32.2015.15311 
emitidos en sede parlamentaria por parlamentarios y parlamentarias en debates, comparecencias, preguntas orales y demás iniciativas que pueden llevarse a cabo en el Parlamento; y por, en segundo lugar, discursos de representante políticos de la administración educativa (se trata de cargos políticos designados por el partido en el Gobierno) fruto de la realización de entrevistas en profundidad y observación.

En el ámbito técnico-administrativo se consideraron para el análisis los discursos extraídos de entrevistas en profundidad realizadas a funcionarios y técnicos especialistas en gestión de la diversidad e interculturalidad en el ámbito educativo; así como, en segundo lugar, los discursos producto de mi participación en calidad de observadora en reuniones y grupos de trabajo desarrollados en las administraciones públicas educativas.

Con respecto a lo que he llamado ámbito normativo, consideré para el análisis textos de leyes y normativas sobre inmigración/extranjería y educación; y por otro lado textos de políticas públicas de educación e integración de la población inmigrante extranjera (planes, programas, proyectos, etc.).

Por último, en lo que he llamado ámbito escolar, los discursos considerados en este caso fueron los obtenidos a través de entrevistas en profundidad y grupos de discusión realizados a y con profesorado y otros profesionales de los centros escolares (orientadores, psicólogos, mediadores, etc.); igualmente mis observaciones en calidad de participante en las dinámicas estándar de los colegios (clases, reuniones, actividades lúdicas/extraescolares, etc.).

Todos estos discursos fueron transformados en texto escrito para poder ser analizados con un programa informático de análisis cualitativo (Nudist Vivo), y a través de cual se aplicaron varias plantillas para facilitar el análisis y la interpretación de la información ${ }^{15}$.

Para responder a las exigencias descritas en el apartado anterior (deconstruir, contextualizar, contrastar emic y etic), diseñé varias plantillas de análisis para sistematizar la información a partir de las siguientes preguntas: a). ¿Qué se dice sobre...?; b). ¿Cómo se dice...?.

La primera pregunta ("Qué se dice sobre...") vendría a responder a un análisis a nivel temático, donde los datos fueron clasificados en tres grandes categorías para trabajar sobre cómo son los procesos de construcción de la alteridad/mismidad, a saber: 1). Caracterización del "nosotros"; 2). Caracterización de los "otros"; y 3). Gestión/tratamiento de la diversidad en la escuela. La segunda pregunta (“¿Cómo se dice...?”) me sirvió para la realización de un análisis de carácter léxico-gramatical, por un lado; y un análisis argumentativo-interpretativo, por otro.

En cuanto a la plantilla de análisis de carácter temático, puede verse una

15 Ello propició poder trabajar con discursos a priori tan diferentes como los discursos parlamentarios y los discursos, por ejemplo, del profesorado una escuela concreta: de todos se consideraron las mismas variables y dimensiones contextuales para la codificación y el análisis. Se elaboraron para ello plantillas con categorías de carácter temático, léxico-gramatical y argumentativo/interpretativo, con lo que poder atender a un proceso de codificación y análisis en los mismos términos - pero que recogiesen las particularidades- de todos los tipos de discurso considerados. 
simplificación de la misma en la Tabla $\mathrm{N}^{\mathrm{o}} 1$ :

\section{Tabla $\mathbf{N}^{0}$ 1. Plantilla de análisis temático}

\begin{tabular}{|c|c|c|c|c|}
\hline \multirow[t]{2}{*}{ 1."Nosotros" } & $\begin{array}{l}\text { A. Población autóctona en general (aspectos que } \\
\text { tienen que ver con la sociedad en su conjunto, } \\
\text { con sus instituciones y sistema educativo) }\end{array}$ & \multirow{4}{*}{ 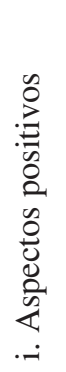 } & \multirow{4}{*}{ 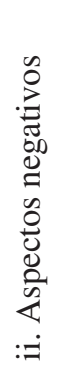 } & \multirow{4}{*}{ 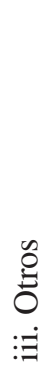 } \\
\hline & B. Alumnado autóctono & & & \\
\hline \multirow[t]{2}{*}{ 2.Los "otros" } & $\begin{array}{l}\text { A. Población inmigrante extranjera en general } \\
\text { (información relativa al fenómeno migratorio, a } \\
\text { los países de origen...) }\end{array}$ & & & \\
\hline & B. Alumnado inmigrante extranjero & & & \\
\hline \multirow{3}{*}{$\begin{array}{l}\text { 3.Gestión/ } \\
\text { tratamiento de } \\
\text { la diversidad en } \\
\text { la escuela }\end{array}$} & \multicolumn{4}{|l|}{ A. Concepto de integración } \\
\hline & \multicolumn{4}{|l|}{ B. Concepto de educación intercultural } \\
\hline & \multicolumn{4}{|l|}{ C. Otros } \\
\hline
\end{tabular}

Fuente: Olmos Alcaraz (2009)

Para evitar que la plantilla me condujese a un análisis reduccionista por estar expresado en términos dicotómicos, se incluyeron varias categorías bajo la rúbrica de "otros", de manera que pudiese con ello rescatar las ausencias (Santos 2009) ${ }^{16}$ y dando por sentado que me encontraría con muchos discursos que no encajasen en el resto de categorías. Además de las ausencias también se consideraron para el análisis las reiteraciones, por lo tanto en el análisis temático se analizó también "de qué no se habla", o "qué temáticas son recurrentes", "a qué aspectos se les concede importancia" y "a qué otros se les resta" y "en relación a qué".

Con la plantilla de análisis de carácter léxico-gramatical traté de registrar qué tipo de lenguaje se usaba de forma más habitual para hablar de inmigración en general y del alumnado inmigrante en particular; pero también cómo se hablaba sobre el "nosotros" cuando se abordaba esta temática. Para ello tuve en cuenta qué figuras retóricas se empleaban (eufemismos, metáforas, estilos gramaticales...) y cuál era el estilo léxico (palabras usadas) en la nominalización y descripción del exogrupo (los "otros") y el endogrupo ("nosotros").

La plantilla de análisis de carácter argumentativo-interpretativo me sirvió

16 El autor habla de una Sociología de las Ausencias para referirse fundamentalmente de un enfoque que pretende traspasar la visión reduccionista que propicia la percepción/construcción del mundo en mitades dicotómicas pensadas conjuntamente como totalidades holísticas. En este sentido retomo su propuesta, en este caso para hacer visible y presente aquello que no encaja fácilmente con lo definido como "positivo" o como "negativo", que no es ni una cosa ni otra o que es ambas a la vez. 
para agrupar y estudiar la información sobre cómo la "sociedad de acogida" estaba entendiendo (describiendo e interpretando) el fenómeno de la inmigración y su presencia en espacios escolares. Las subcategorías consideradas fueron: 1). Problemáticas asociadas y ventajas percibidas de la inmigración y su presencia en la escuela; 2). Modelos interpretativos de esta realidad (discursos paternalistas, economicistas, alarmistas, culturalistas... que no definen claramente el fenómeno migratorio en términos de ventajas o inconvenientes -léase para la sociedad receptora, casi siempre-); y 3). Estrategias discursivas de construcción de la diferencia: formas de argumentar para convencer y crear consentimiento y formas que tratan de modificar la realidad social propiciando que sea interpretada de una manera determinada, sin dejar opción a alternativas. Véase a continuación parte de la plantilla para el análisis argumentativointerpretativo, concretamente la sección de la misma que se refiere a las estrategias discursivas de construcción de la diferencia:

\section{Tabla $N^{\circ}$ 2. Plantilla de análisis argumentativo-interpretativo: estrategias} discursivas de construcción de la diferencia

\begin{tabular}{|l|l|}
\hline 1. Expresión de estereotipos y prejuicios de manera tolerable. \\
\hline 2. Vinculación recurrente de temas. \\
\hline 3. Silenciar y/o deslegitimar otros discursos. \\
\hline \multirow{5}{*}{ 4. Eludir responsabilidades. } & $\begin{array}{l}\text { A. Aludiendo a que "todos piensan/actúan igual } \\
\text { que yo". }\end{array}$ \\
\cline { 2 - 3 } & $\begin{array}{l}\text { B. Aludiendo a que son los "otros" los que } \\
\text { piensan/actúan de una determinada manera y } \\
\text { no yo. }\end{array}$ \\
\cline { 2 - 3 } & $\begin{array}{l}\text { C. Creando un chivo expiatorio (no sólo } \\
\text { eluden responsabilidades sobre pensamientos } \\
\text { y acciones sino que se busca a otro culpable } \\
\text { que cargue con ellas)- Justificando discursos y } \\
\text { acciones por motivos excepcionales. }\end{array}$ \\
\cline { 2 - 3 } 5. Búsqueda de credibilidad. & $\begin{array}{l}\text { D. Invisibilizando a los agentes de discursos y } \\
\text { acciones. }\end{array}$ \\
\hline $\begin{array}{l}\text { A. Apoyándose en discursos ya legitimados (de } \\
\text { otras personas, de leyes y otros instrumentos } \\
\text { normativos, de investigaciones...). }\end{array}$ \\
\cline { 2 - 2 } & B. Naturalizando discursos y acciones. \\
\hline
\end{tabular}

Fuente: Olmos Alcaraz (2009) 
Estos instrumentos me permitieron un análisis de los discursos recogidos y producidos a lo largo de mi actividad de trabajo de campo en los términos descritos en los apartados anteriores. Los mismos fueron diseñados atendiendo a los aportes que el ACD realiza para el análisis y las posibilidades que brinda la Etnografía en tanto que metodología que propicia una relación cercana entre sujetos investigados e investigadores.

Y termino este apartado con un ejemplo. Una de las estrategias discursivas de construcción de la diferencia detectada a lo largo de mi investigación fue la que denomino como "Expresión de estereotipos y prejuicios de manera tolerable". Esta estrategia es una característica de los discursos sobre la inmigración en el contexto estudiado, que contribuye de forma muy importante a la creación de relaciones de alteridad. Las puesta en práctica de las herramientas que proporciona el ACD me permitió analizar con mucho detalle y profundidad distintas formas en que dicha estrategia podría presentarte tanto en políticos, técnicos y funcionarios de la administración, como profesorado. Pero para poder establecer relaciones analíticas entre la ocurrencia de dicha estrategia en los distintos agentes estudiados fue esencial poder contextualizar (en los términos esbozados en el apartado anterior) sus discursos. Ello fue posible gracias a mi trabajo de campo etnográfico, que me posibilitó para funcionar con distintos tipos de datos: emic y etic; y me posibilitó observar y participar en los espacios de producción de los discursos. Es decir, la combinación de ACD y etnografía facilitó tener una visión más profunda para desvelar los significados y funcionamiento que puede tener un prejuicio expresado de manera tolerable. Así pude ver que estereotipos como que "los inmigrantes son unos delincuentes porque no encuentran trabajo y de algo tienen que vivir", expresados por un político en sede parlamentaria podía significar una acusación al Gobierno de no estar implementando las políticas migratorias adecuadas; mientras que para un profesor podía no ser más que una evidencia de que la inmigración es un fenómeno "construido socialmente como un problema", cuando la misma lógica discursiva no era aplicada con respecto a los "autóctonos".

\section{CONCLUSIONES: HACIA LA COMPLEMENTARIEDAD METODOLÓGICA EN CIENCIAS SOCIALES}

En este artículo he mostrado el diseño metodológico seguido en la realización de mi investigación de tesis doctoral, donde el objeto teórico de estudios eran las relaciones de alteridad construidas hacia la población inmigrante en España. El foco ha sido puesto en explicitar los ejes epistemológicos sobre los que ha girado la investigación, tratando de poner en valor los aportes de un trabajo con vocación inter, multi y trans-disciplinar. Dicho lo cual, a modo de reflexiones conclusivas de mi trabajo, destaco las siguientes ideas.

En primer lugar, debo reconocer que esta forma de trabajar es más costosa que otras formas de hacer investigación. Los procesos son más complejos 
y complicados de poner en marcha, porque no se trata de "trasplantar" metodologías que ya se ha demostrado que son exitosas a un objeto en particular, sino que -en cierto modo- se trata de experimentar en el plano metodológico. Sin embargo, también entiendo que los aportes conseguidos son mayores, porque no solo se obtienen resultados de investigación en el plano teórico, o empírico, sino que los procesos seguidos son en sí mismos resultados de investigación. Se ha podido establecer una relación dialógica entre distintas métodos, técnicas y enfoques (con el uso de unos se ha logrado llenar el hueco que dejan los límites de otros), algo no tan habitual dentro de Ciencias Sociales, entre otras cuestiones, por los recelos propios existentes entre la Antropología, la Sociología, la Psicología Social, la Lingüística, etc.

En segundo lugar me gustaría reflexionar acerca de si mi investigación puede situarse dentro de lo que se conoce como triangulación metodológica, o ha conseguido ir más allá. Mi posición al respecto es que se ha trascendido el plano estrictamente metodológico, a pesar de estar en este texto describiendo justamente dicho proceso, porque este diálogo ha forzado en sí mismo el diálogo entre disciplinas. Por otro lado, rescato que dicho enfoque ha impregnado todo el trabajo de investigación, no solo la recogida/producción de datos, sino que en el resto de fases -la conformación del marco teórico, y el análisis, por supuesto- se ha seguido el posicionamiento de la complementariedad e intercambio.

Por último señalar también, como no, algunos de los retos que considero quedan abiertos para el futuro dentro de mi trabajo de investigación, que entiendo en el centro de algunos debates actuales en Ciencias Sociales. En este sentido, reflexionar sobre la posición de los/as propios/as investigadores/as en el quehacer científico, en tanto que promotores de diálogos no estrictamente disciplinares, ya que estos pueden estar obviando o relegando a un segundo plano la implicación práctica para resolver -y explicar- los problemas sociales sobre los que trabajamos. Y por otro lado, poner sobre la mesa el debate sobre la necesidad de una "descolonización del conocimiento" (Santos 2009; Grosfoguel 2011), que indica como uno de sus postulados -aunque no el más importantela reflexión sobre los problemas existentes dentro de la academia al vernos obligados y constreñidos en nuestro empeño por ubicarnos en compartimentos estancos, en muchas ocasiones, limitadores frente a la posibilidad de trabajar de manera inter, multi y trans-disciplinar.

\section{BIBLIOGRAFÍA}

ALVIRA MARTÍN, F. (1983): "Perspectiva cualitativa-perspectiva cuantitativa en la metodología sociológica”, Revista Española de Investigación Sociológica, 22, pp. 53-76.

BERGER, P. y LUCKMANN, T. (2006): La construcción social de la realidad, Buenos Aires, Amorrortu.

BURR, V. (2003): Social Constructionism, Londres, Routledge. 
CAPELlÁN DE TORO, L., GARCÍA CASTAÑO, F.J., OLMOS ALCARAZ, A. y RUBIO GÓMEZ, M. (2013): "Sobre agrupamiento, concentración, segregación o guetización escolar: claves para un análisis interpretativo de tales situaciones y procesos", Avances en Supervisión Educativa, 18, pp. 1-27.

CHARAUDEAU, P. y MAINGUENEAU, D. (2005): Diccionario de análisis del discurso, Madrid, Amorrortu Editores.

DERRIDA, J. (1975): La diseminación, Madrid, Editorial Fundamentos.

DIETZ, G. (2012): "Reflexividad y diálogo en etnografía colaborativa: el acompañamiento etnográfico de una institución educativa "intercultural" mexicana", Revista de Antropología Social, 21, pp. 63-91.

DIETZ, G. y MATEOS CORTES, L. S. (2010): "La etnografía reflexiva en el acompañamiento de procesos de interculturalidad educativa: un ejemplo veracruzano", Cuicuilco, 48, pp. 107-131.

FAIRCLOUGH, N. (1998): "Propuestas para un nuevo programa de investigación en el análisis crítico del discurso", en Poder-decir o el poder de los discursos, Madrid, The British Council, Arrecife y UA Ediciones, pp. 35-54.

(2001): Languaje and power, Harlow, Longman.

FOUCAULT, M. (1970): La arqueología del saber, México, Siglo XXI. (2002): El orden del discurso, Barcelona, Tuquets.

GARCÍA GARCÍA, J. L. (1996): "El análisis del discurso en la Antropología Social”, en Etnolinguística y análisis del discurso, Zaragoza, Instituto Aragonés de Antropología y Federación de Asociaciones de Antropología del Estado Español, pp. 9-11. (2000): "Informar y narrar: el análisis de los discursos en las investigaciones de campo", Revista de Antropología Social, 9, pp. 75-104.

GARCÍA CASTAÑO, F. J., GRANADOS MARTÍNEZ, A. y PULIDO MOYANO, R. (1999): "Reflexiones en distintos ámbitos de construcción de la diferencia", en Lecturas para educación intercultural, Madrid, Trotta, pp. 15-46.

GEERTZ, C. (1973): La interpretación de las culturas, Barcelona, Gedisa.

GÓMEZ, E. (1995): "La evolución del concepto de etnografía", en Etnografía. Metodología cualitativa en la investigación sociocultural, Terrassa, Alfaomega/ Marcombo, pp. 21-44.

GONZÁLEZ ECHEVARRÍA, A. (2009): La dicotomía emic/etic. Historia de una confusión, Barcelona, Anthropos.

GROSFOGUEL, R. (2006): "La descolonización de la economía política y los estudios postcoloniales. Transmodernidad, pensamiento fronterizo y colonialidad global", Tabula Rasa, 4, p. 17-48.

(2011): "La descolonización del conocimiento: diálogo crítico entre la visión descolonial de Frantz Fanon y la sociología descolonial de Boaventura de Sousa Santos", en Formas-Otras: saber, nombrar, narrar, hacer, Barcelona, CIDOB, pp. 97-108.

(2012): Sujetos coloniales: una perspectiva global de las migraciones caribeñas, Quito, Abya Yala.

GUBER, R. (2010): "La autonomía etnográfica. El trabajo de campo de los antropólogos sociales argentinos entre 1965 y 1975”, Antípoda. Revista de antropología y arqueología, 11, pp. 189-215.

HAMMERSLEY, M. y ATKINSON, P. (2001): Etnografía. Métodos de investigación, Barcelona, Paidós.

HARAWAY, D. (1995): Ciencia, cyborgs y mujeres: la reinvención de la naturaleza, Madrid, Ediciones Cátedra. 
HINE, C. (2004): Etnografía virtual, Barcelona, UOC.

JESÚS ORTECHO, M. (2013): Estrategias discursivas emergentes empleadas en Proyectos Intersectoriales de orientación "social". Caso: Campaña Ningún Hogar Pobre en Argentina, Córdoba, Editorial del Centro de Estudios Avanzados.

LASSITER, L. E. (2005): The Chicago Guide to Collaborative Ethnography, Chicago, University of Chicago Prees.

MALINOWSKI, B. (1993): "Introducción: objeto, método y finalidad de esta investigación", en Lecturas de Antropología Social y Cultural. La cultura y las culturas, Madrid, UNED, pp. 89-112.

MARCUS, G. (1995): "Ethnography in/of the World System: The Emergence of MultiSited Ethnography", Annual Review of Anthropology, 24, pp. 95-117. (2008): "El o los fines de la etnografía: del desorden de lo experimental al desorden barroco", Revista de Antropología Social, 17, pp. 27-48.

MARTÍN ROJO, L., PARDO, M. L. y WHITTAKER, R. (1998): "El análisis crítico del discurso: una mirada indisciplinada", en Poder-decir o el poder de los discursos, Madrid, The British Council, Arrecife y UA Ediciones, pp. 9-33.

OLMOS ALCARAZ, A. (2009): La población inmigrante extranjera y la construcción de la diferencia. Discursos de alteridad en el sistema educativo andaluz (Tesis doctoral. Departamento de Antropología Social), Granada, Universidad de Granada.

(2010): "Construcción discursiva del alumno inmigrante extranjero en Andalucía: el otro en nuestras escuelas", Revista de Educación, 353, pp. 469-493.

(2012): "Cuando migrar se convierte en estigma: un estudio sobre construcción de alteridad hacia la población inmigrante extranjera en la escuela", Imagonautas. Revista Interdisciplinaria Sobre Imaginarios Sociales, 1 (2), pp. 62-85.

OLMOS ALCARAZ, A. y RUBIO GÓMEZ, M. (2013): "Corporalidad del «buen estudiante»: representaciones de género, «raza», etnia y clase social en la escuela española", Revista Iberoamericana de Educación, 62, pp. 163-179.

POTTER, J. (1998): La representación de la realidad. Discurso, retórica y construcción social, Barcelona, Paidós.

QUIJANO, A. (2000): “Colonialidad del poder y clasificación social”, Journal of WorldSystem Research, 2, pp. 342-386.

SCHEPER-HUGHES, N. (1995): "The Primacy of the Ethical: Propositions for a Militant Anthropology", Current Anthropology, 36 (3), pp. 409-440.

TODOROV, T. (1991): Nosotros y los otros. Reflexión sobre la diversidad humana, Madrid, Siglo veintiuno.

URIBE MALLARINO, C. (2012): “Interdisciplinariedad en investigación: ¿colaboración, cruce o superación de las disciplinas?”, Universitas humanística, 73, pp. 147-172.

VAN DIJK, T. A. (1993): Elite discourse and racism, California, Newbury Park, SAGE. (1997): Racismo y análisis crítico de los medios, Barcelona, Paidós. (1999a): "El análisis crítico del discurso", Anthropos, 189, pp. 23-26. (1999b): Ideología. Una aproximación multidisciplinaria, Barcelona, Gedisa.

(2003): Ideología y discurso, Barcelona, Ariel Lingüística.

(2008): Discourse and Power. Contributions to Critical Discourse Studies, Houndsmills, Palgrave MacMillan. (2009):Discurso y poder, Barcelona, Gedisa.

(2010): "Discurso, conocimiento, poder y política. Hacia un análisis crítico epistémico del discurso", Revista de Investigación Lingüística, 13, pp. 167-215. 
VASILACHIS DE GIALDINO, I. (1997): La construcción de representaciones sociales. Discurso político y prensa escrita. Un análisis sociológico, jurídico y lingüístico, Barcelona, Gedisa.

VELASCO, HONORIO y DÍAZ DE RADA, A. (1999): La lógica de la investigación etnográfica. Un modelo de trabajo para etnógrafos de la escuela, Madrid, Trotta.

WALSH, C. (2007): “¿Son posibles unas ciencias sociales/culturales otras? Reflexiones en torno a las epistemologías decoloniales”, Nómadas, 26, pp. 102-113.

WERNER, OSWALD y SHOEPFLE, M. (1993): "Cuestiones epistemológicas", en Lecturas de Antropología Social y Cultural. La cultura y las culturas, Madrid, UNED, pp. 113-181.

WILCOX, K. (2005): "La etnografía como una metodología y su aplicación al estudio de la escuela. Una revisión”, en Lecturas de Antropología para educadores. El ámbito de la antropología de la educación y de la etnografía escolar, Madrid, Trotta, pp. 95-126.

WODAK, R. y MATOUSCHEK, B. (1998). "Se trata de gente que con sólo mirarla se adivina su origen: análisis crítico del discurso y el estudio del neo-racismo en la Austria contemporánea", en Poder-decir o el poder de los discursos, Madrid, The British Council, Arrecife y UA Ediciones. Pp. 55-92.

WOLCOTT, H. (2005): "Sobre la intención etnográfica", en Lecturas de Antropología para educadores. El ámbito de la antropología de la educación y de la etnografía escolar, Madrid, Trotta, pp. 127-144. 
